\title{
Recombination processes in unintentionally doped GaTe single crystals
}

\author{
A. Zubiaga and J. A. García ${ }^{a}$ \\ Departamento de Física Aplicada II, Facultad de Ciencias, Universidad del País Vasco, \\ Lejona (Vizcaya), Spain \\ F. Plazaola \\ Departamento de Electricidad y Electrónica, Facultad de Ciencias, Universidad del País Vasco, \\ Lejona (Vizcaya), Spain \\ V. Muñoz-Sanjosé and M. C. Martínez-Tomás \\ Departamento de Física Aplicada and Instituto de Ciencia de Materiales de la Universitat de València \\ (ICMUV), C/Doctor Moliner No. 50, E-46100 Burjassot (València), Spain
}

(Received 25 April 2002; accepted 27 September 2002)

\begin{abstract}
Emission spectra of GaTe single crystals in the range of 1.90-1.38 eV have been analyzed at different temperatures and excitation intensities by photoluminescence, photoluminescence excitation, and selective photoluminescence. A decrease in band gap energy with an increase in temperature was obtained from the redshift of the free exciton recombination peak. The energy of longitudinal optical phonons was found to be $14 \pm 1 \mathrm{meV}$. A value of $1.796 \pm 0.001 \mathrm{eV}$ for the band gap at $10 \mathrm{~K}$ was determined, and the bound exciton energy was found to be $18 \pm 0.3 \mathrm{meV}$. The activation energy of the thermal quenching of the main recombination peaks and of the ones relating to the ionization energy of impurities and defects was analyzed. The results obtained show the existence of two acceptor levels with ionization energies of $110 \pm 5$ and $150 \pm 5 \mathrm{meV}$, respectively, and one donor level with an ionization energy of $75 \pm 5 \mathrm{meV}$. The study of chemical composition by inductively coupled plasma-optical emission spectroscopy and X-ray energy dispersion spectroscopy shows the existence of $\mathrm{Na}, \mathrm{Li}$, and $\mathrm{Si}$. Sodium and lithium impurities could be associated with acceptor levels at gallium substitutional sites, and silicon ones with a donor level at Ga sites, whose vacancies can also be involved in these electronic levels. (C) 2002 American Institute of Physics. [DOI: 10.1063/1.1523144]
\end{abstract}

\section{INTRODUCTION}

Due to strong excitonic emission, some III-VI compound semiconductors are of interest because of their potential applications as photon detectors, solid state lasers, and optoelectronic devices. Some of these compounds, such as $\mathrm{GaS}, \mathrm{GaSe}$, and GaTe, are layered and this feature results in anisotropic properties. Bonds within the layers are mainly covalent and the layers are bound by van der Waals forces. Among these compounds GaTe is the least studied. It has a monoclinical layered structure with $C_{2 / m}$ space. Each primitive cell contains 6 molecules and the unit cell contains 12 molecules and 108 valence electrons. ${ }^{1,2}$ While other III-VI semiconductors crystallize in a four-sheet intralayer stacking pattern and all the $\mathrm{Ga}-\mathrm{Ga}$ bonds are perpendicular to the layer plane, GaTe has only two thirds of the $\mathrm{Ga}-\mathrm{Ga}$ bonds perpendicular to the layer planes while the others lie in the layer plane. ${ }^{3}$ These bonds form chains of twofold rotational symmetry inside the layers along the twofold rotational symmetry axis ${ }^{1}$ and the optical and crystallographic properties are different with regard to the other III-VI compounds. For a detailed description see Refs. 2 and 3.

Some efforts have been made to study the anisotropic structural and electronic transport properties of GaTe, ${ }^{4,5}$ its refractive index, ${ }^{6}$ and its physical properties under pressure. ${ }^{7}$

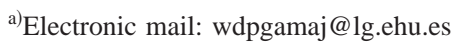

Optical measurements have also been done: Camassel et al. ${ }^{8}$ performed absorption experiments at the liquid helium temperature and they found strong excitonic absorption. Two types of free exciton recombination at $1.779(n=1)$ and $1.791 \mathrm{eV}(n=2)$ were clearly visible and the Rydberg energy was found to be $16 \mathrm{meV}$. Other authors ${ }^{9-14}$ have studied the structure of free excitonic recombination in photoluminescence (PL) experiments. However, very little work has been done on optical recombination apart from that due to free excitons.

By measuring time resolved photoluminescence at $4 \mathrm{~K}$ Taylor and Ryan ${ }^{15}$ found a well resolved peak at $1.778 \mathrm{eV}$ and a broad band extending from 1.760 to $1.660 \mathrm{eV}$ in the spectrum of $p$-GaTe. These two bands were attributed to the recombination of free excitons and excitons bound to a neutral acceptor, respectively. Shigetomi et al. ${ }^{16}$ working at $97 \mathrm{~K}$ obtained a PL spectrum with two emission bands at 1.76 and $1.59 \mathrm{eV}$. The $1.76 \mathrm{eV}$ band was explained by emission due to free exciton recombination and the $1.59 \mathrm{eV}$ band was assumed to be related to the transition from a donor level to an acceptor level located $150 \mathrm{meV}$ above the valence band. The position of the acceptor level in the band gap was inferred from the thermal activation energy of the $1.59 \mathrm{eV}$ peak and it agrees with an acceptor energy level (145 meV) previously obtained by Hall effect measurements. ${ }^{17}$ More recently Guder et al. ${ }^{18}$ found three emission bands at $10 \mathrm{~K}$ located at 1.781 ( $A$ band), 1.735 ( $B$ band), and $1.575 \mathrm{eV}$ ( $C$ band). They 
related these three bands to the recombination of free excitons, bound excitons, and donor-acceptor pairs (DAPs), respectively. From calculation of the activation energy (56 meV) of the $C$ band, they concluded that this band was DAP recombination from a donor level at $170 \mathrm{meV}$ to an acceptor level at $56 \mathrm{meV}$. For the $B$ band they obtained an activation energy of $46 \mathrm{meV}$.

It seems that the $1.59 \mathrm{eV}$ emission observed by Shigetomi et al. ${ }^{16}$ and the $1.575 \mathrm{eV}$ emission obtained by Guder et $a l .{ }^{18}$ are due to the same DAP recombination. However, the distribution of levels involved in the recombination differs between the two authors. On the other hand, the broad band observed by Taylor and Ryan ${ }^{15}$ in the $1.760-1.660 \mathrm{eV}$ region may be related to the narrower band observed by Guder et al. ${ }^{18}$ at $1.735 \mathrm{eV}$. Due to the discrepancies in the above results and the lack of more information on the PL of GaTe it has been very difficult to infer the distribution of levels near the band gap and the recombination processes in this semiconductor.

In order to clarify the radiative recombination process in unintentionatelly doped $p$-GaTe, we have studied the PL of GaTe single crystals in the 1.9-1.4 eV range and also used photoluminescence excitation and selective photoluminescence experiments. Variation of the PL with the power of the excitation light and temperature has also been studied. The results are complemented by inductively coupled plasma optical emission spectroscopy analysis and scanning electron microscopy observations of the samples in an apparatus with an x-ray energy dispersion analysis system.

\section{EXPERIMENT}

Low temperature PL measurements were performed using the laser port of a CD900 spectrometer system from Edinburgh Instruments that has a R955 photomultiplier in a Peltier cooled housing. The samples were mounted on the cold finger of a closed-cycle He cryostat in the $10-300 \mathrm{~K}$ temperature range and they were excited by the $488 \mathrm{~nm}$ line of a multiline ion-argon laser which can generate up to $1 \mathrm{~W}$ of power for this line. For photoluminescence excitation and selective photoluminescence experiments, the excitation source was a $450 \mathrm{~W}$ Xe lamp attached to the CD900 excitation monochromator. The spectral resolution was $1 \mathrm{meV}$ for PL measurements and $2 \mathrm{meV}$ for photoluminescence excitation and selective photoluminescence measurements. The scanning electron microscopy inspections were accomplished using a JEOL 6400 with an x-ray dispersion analysis system, Pentafet Link, from Oxford Instruments. Quantitative composition measurements of the samples were made using a Thermo ARL 3140 inductively coupled plasmaoptical electron spectrometer (ICP-OES). Ingots of GaTe were grown by the Bridgman-Stockbarger method from a polycrystalline material previously synthesized from high purity $(5 \mathrm{~N})$ elemental gallium and tellurium. The ingots were constituted of some big monocrystalline regions from which the single crystals analyzed here were cleaved. Before the experimental study the samples were freshly cleaved and quickly mounted in the cryostat to avoid surface oxidation.

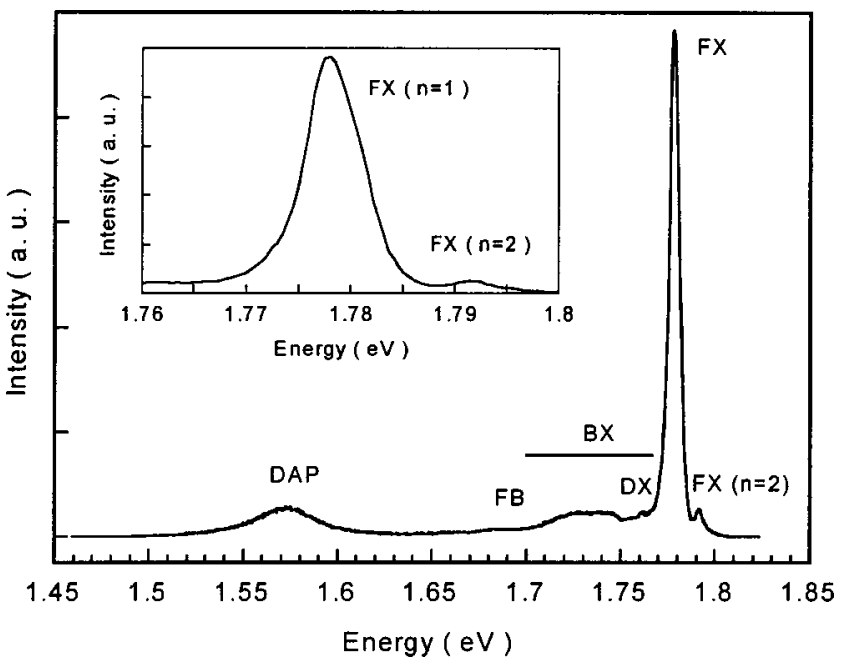

FIG. 1. PL spectrum of a monocrystalline GaTe sample at $10 \mathrm{~K}$ excited with $2.54 \mathrm{eV}$ energy which shows the recombination peaks measured in the samples.

The orientation of the samples was verified by x-ray diffraction. All the samples have their surfaces perpendicular to the twofold rotational symmetry axis. ${ }^{1,9}$

\section{RESULTS AND DISCUSSION}

The spectrum in Fig. 1 shows PL recombination peaks when exciting with $2.54 \mathrm{eV}$ laser radiation. Next we will describe the more relevant emissions and assign them to different recombination processes. These assignments will be verified in the discussion of experimental results that will be subsequently analyzed and discussed. In Fig. 1 the more relevant emissions are indicated. The free exciton peak $(F X)$ dominates the spectrum and the $n=2$ excited state of $F X$ at $1.792 \mathrm{eV}$ and the $F X$ emission at $1.776 \mathrm{eV}$ can be observed. In the range between 1.76 and $1.70 \mathrm{eV}$ a bound exciton $(B X)$ broad band can be seen. This band is composed of a peak at $1.765 \mathrm{eV}$, due to the recombination of an exciton at a donor level $(D, X)$, and two emissions at 1.720 and $1.730 \mathrm{eV}$. We will see later that those are associated with the recombination of excitons at two different acceptor levels, which we will denote $\left(A_{2}, X\right)$ and $\left(A_{1}, X\right)$. On the low energy side of the $B X$ band, a free to bond recombination $(F B)$ at $1.685 \mathrm{eV}$ appears. Finally, there is also broad donor-acceptor pair emission centered at $1.57 \mathrm{eV}$. The relative intensities of the peaks change for different samples and different regions within a sample. This behavior can be observed in Fig. 2, where spectra for three different regions of the same sample are shown. It can be seen that the main difference lies in the shape of the bound exciton band, $B X$.

\section{A. Free exciton luminescence}

From the position of the $F X_{n=1}$ and $F X_{n=2}$ peaks at 10 $\mathrm{K}$, we can determine the exciton binding energy and the gap energy at this temperature using the relation

$$
E_{n}=E_{g}-R_{x} / n^{2} \text {. }
$$

The values obtained are $R_{x}=18.0 \pm 0.3 \mathrm{meV}$ and $E_{g}$ $=1.796 \pm 0.001 \mathrm{eV}$. Following variation of the energy of the 


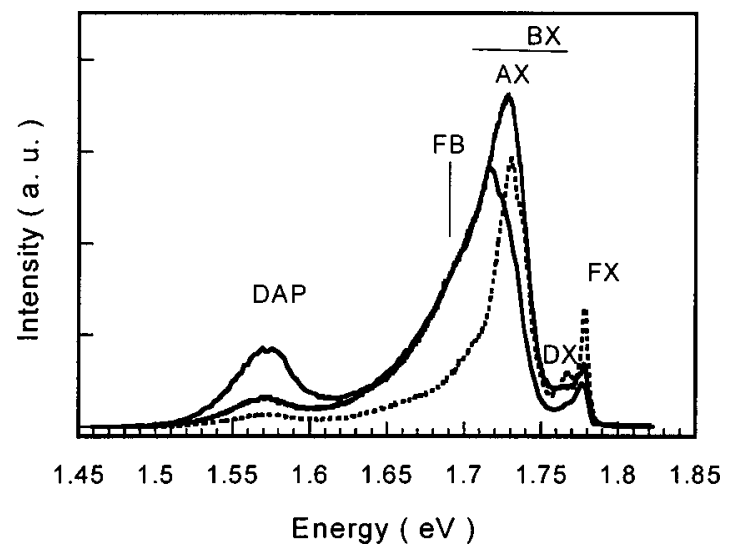

FIG. 2. PL spectra from three different regions of a GaTe sample at $10 \mathrm{~K}$ excited with $2.54 \mathrm{eV}$ energy.

$F X$ peak according to the temperature, while exciting with the $488 \mathrm{~nm}$ line $(2.54 \mathrm{eV})$ of an ion-argon laser, the change in the energy gap with the temperature can be established for temperatures up to $200 \mathrm{~K}$. Figure 3 shows the free exciton peak energy as a function of the temperature and the numerical fit based on the semiempirical model of O'Donnell and Chen. ${ }^{19}$ This numerical fit can be described by

$$
E_{g}(T)=E_{g}(0)-S\langle\hbar \omega\rangle\left[\operatorname{coth}\left(\frac{\langle\hbar \omega\rangle}{2 K_{B} T}\right)-1\right],
$$

and presents a better fit than that described by the empirical model of Varshni. ${ }^{20}$ Using the O'Donnell and Chen model, the dimensionless coupling constant $S$, based on the vibronic model of Huang and Rhys, comes out at 4.0 and the average phonon energy involved in the decrease of the gap is 14 $\pm 1 \mathrm{meV}$.

The longitudinal optical (LO) phonons in GaTe, determined by Wan et al. ${ }^{10}$ from PL measurements up to $40 \mathrm{~K}$ of the full width at half maximum of the free exciton peak, have an energy of $14 \mathrm{meV}$, which agrees with the value obtained in the above fit. Moreover, this value is the same as the energy proposed by Camassel et al. in Ref. 8. In order to fit the temperature dependence of the $n=1$ excitonic line, Camassel and co-workers assumed a strong interaction with a $14 \mathrm{meV}$ phonon which was the average of the 13.6 and 14.3

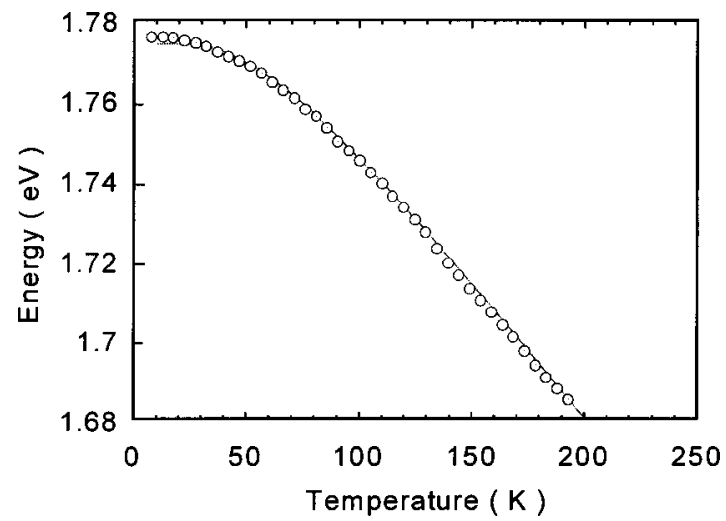

FIG. 3. Experimental values of the free exciton peak position as a function of the temperature. The solid line is the numerical fit based on the O'Donnell-Chen empirical model (Ref. 19).

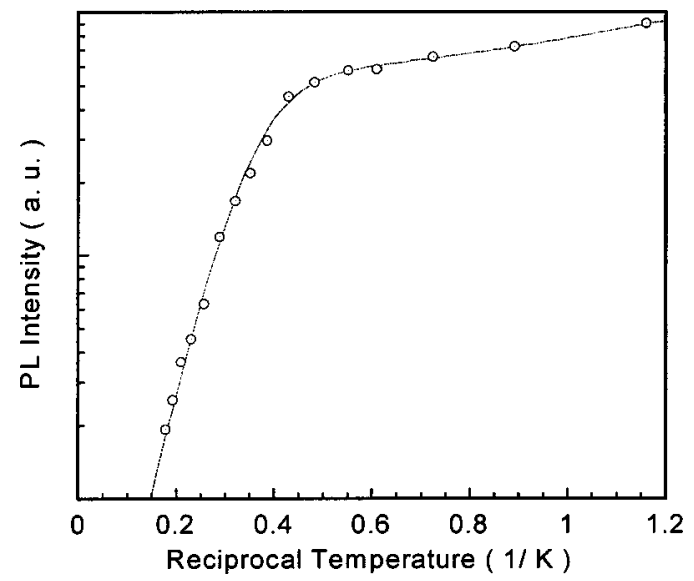

FIG. 4. Intensity quenching of the free excitons as a function of the temperature using a two-step quenching process.

meV phonon energies associated with the phonon frequencies of 110 and $115 \mathrm{~cm}^{-1}$ obtained in the lattice dynamic study in Ref. 21. These results indicate that, in the temperature range measured, $\mathrm{LO}$ phonon-electron interaction is the dominant contribution to the variation of the band gap. The value of the dimensionless parameter $S$ of the O'DonnellChen fit gives an idea of the number of phonons involved in the interaction, i.e., the magnitude of phonon coupling with electrons, and its value in this work was within the typical range for compound semiconductors. ${ }^{19}$ By adding the calculated exciton binding energy to the free exciton recombination energy, the energy band gap of GaTe over the 10-200 K range can be determined. The extrapolated values obtained for the energy band gap at 0 and $300 \mathrm{~K}$ were 1.796 and $1.641 \pm 0.001 \mathrm{eV}$, respectively.

The variation in PL intensity of the free exciton recombination as a function of the temperature is shown in Fig. 4. The experimental observation can be fitted by considering a two-step quenching process described by

$$
\begin{aligned}
I_{\mathrm{PL}}= & \frac{I_{1}}{1+C_{1} \sqrt{T^{3}} \exp \left(-A_{1} / k_{B} T\right)} \\
& +\frac{I_{2}}{1+C_{2} \sqrt{T^{3}} \exp \left(-A_{2} / k_{B} T\right)} .
\end{aligned}
$$

The values for activation energies $A_{1}$ and $A_{2}$ are 3 and $17 \mathrm{meV}$, respectively. Krustok et al. ${ }^{22}$ working on CdTe PL data, established that the low value of the $A_{1}$ parameter, obtained using a two-exponential fitting equation, may just be an artifact without genuine physical significance. Rather than being due to a true difference in energy level at the low temperature end, the reduction of the PL intensity with an increase in temperature seems to be related to the approximate $T^{-2}$ temperature dependence of the cross section. Without any further evidence, this could be one interpretation for the low-temperature parameter $\left(A_{1}\right)$ obtained.

The high temperature activation energy, $A_{2}=17 \mathrm{meV}$, is in good agreement with the $18 \mathrm{meV}$ of the previously mea- 


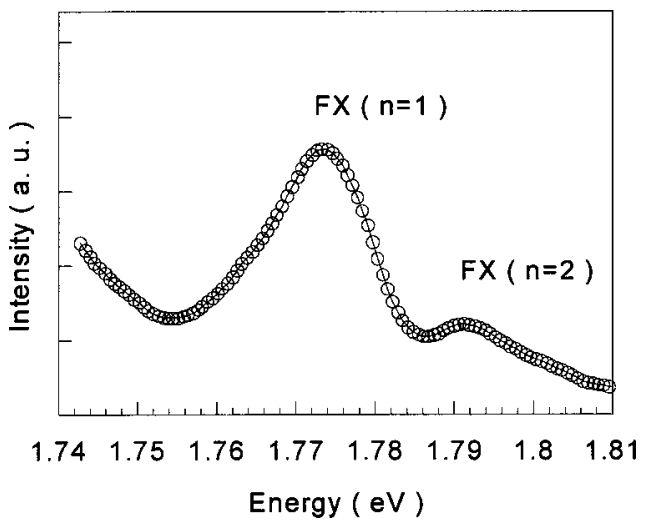

FIG. 5. Photoluminescence excitation spectra at $10 \mathrm{~K}$ for the $B X$ band, measured at an emission energy of $1.73 \mathrm{eV}$.

sured binding energy. This indicates that the quenching process of $F X$ emission is due to thermal dissociation of the bound electron-hole pair.

\section{B. Bound excitons and free-to-bound emissions}

In most of the samples measured the PL spectrum at low temperature is dominated by bound exciton emission $B X$. The intensity of this band changes from one sample to another and even in different zones of the same sample as shown in Fig. 2. The results obtained in the present work suggest that this band is mainly composed of emission lines at $1.730\left(A_{1}, X\right)$ and $1.720 \mathrm{eV}\left(A_{2}, X\right)$, associated with the recombination of excitons at two different acceptor levels. Looking for confirmation, several experiments at different temperatures, excitation powers, and excitation energies were performed.

The photoluminescence excitation experiments for the $B X$ band confirm the excitonic character of this band. Figure 5 shows a photoluminescence excitation spectrum for a selected emission energy of $1.73 \mathrm{eV}$ at the $B X$ band. It can be seen that the $B X$ emission reaches its maximum intensity when the excitation energy is that of the $F X$ peak. Consequently the $B X$ band is closely related to the resonant creation of excitons.

Figure 6 gives the variation according to the temperature

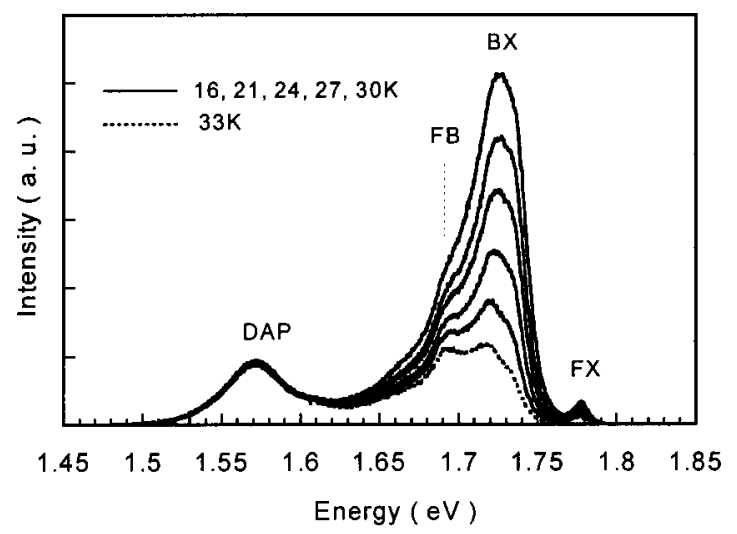

FIG. 6. Emission spectra of a GaTe sample for different temperatures. All spectra are on the same scale.

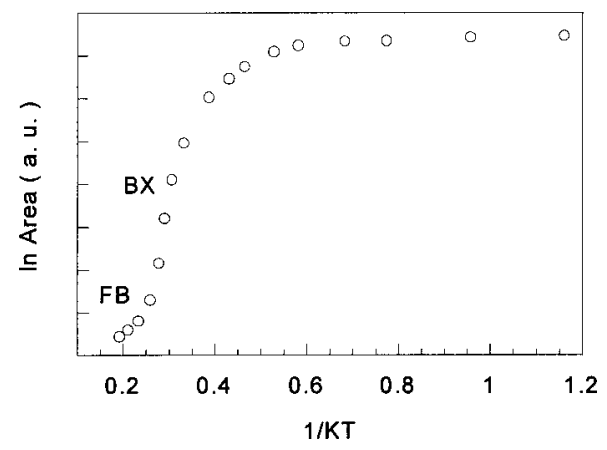

FIG. 7. Temperature dependence of the logarithmic emission-line area for the $B X$ broad band.

of the PL spectra of a sample that shows a broad $B X$ band. As the temperature increases, the high energy side of the $B X$ emission decreases, remaining a $F B$ emission that disappears at higher temperatures.

The dependence of the logarithm of PL intensity for the $B X$ band as a function of $1 / k T$ (Fig. 7) shows the structure of this band. The coexistence of two different slopes in the high temperature region of this graph can be seen. These slopes correspond to at least two quenching processes, the one at lower temperature probably due to bound exciton recombination and the other (that at higher temperature) due to $F B$ recombination. The structure of the $B X$ band can also be observed by analyzing its variation as a function of the excitation power of the $2.54 \mathrm{eV}$ laser line (Fig. 8). In these experiments the behavior of the broad $B X$ band for two different ranges of excitation power can be distinguished. Initially, in the range up to $1 \mathrm{~kW} / \mathrm{cm}^{2}$, the intensity of all the components of the $B X$ emission increases with an increase in excitation energy. For higher powers, the band can be separated into different emission components, 1.72 and $1.73 \mathrm{eV}$, which change their emission intensities and move toward lower energies as the excitation intensity increases. For this excitation range the $F B$ emission dominates the spectra. This behavior can be understood if we take into account that the density of free excitons increases for high excitation intensi-

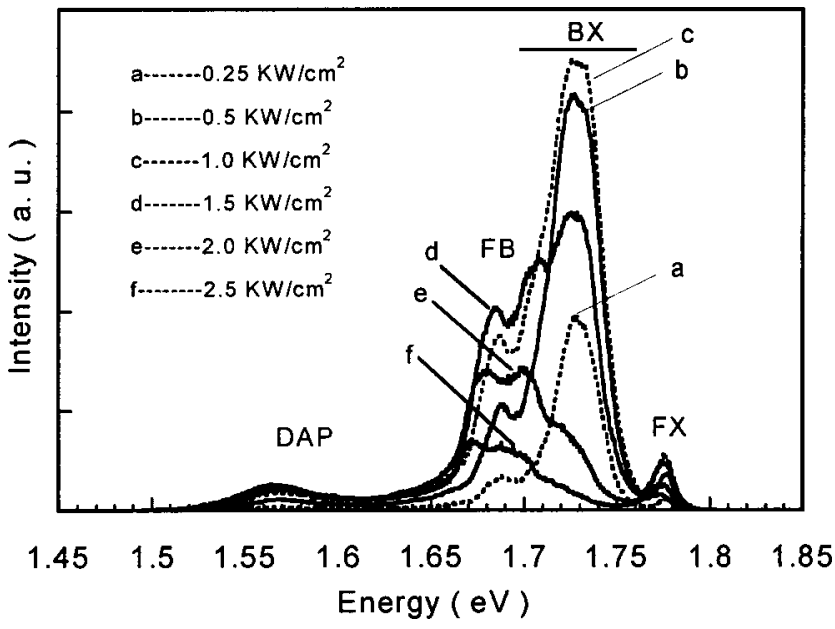

FIG. 8. Emission spectra of a GaTe sample at $2.54 \mathrm{eV}$ for different excitation powers. All spectra are on the same scale. 


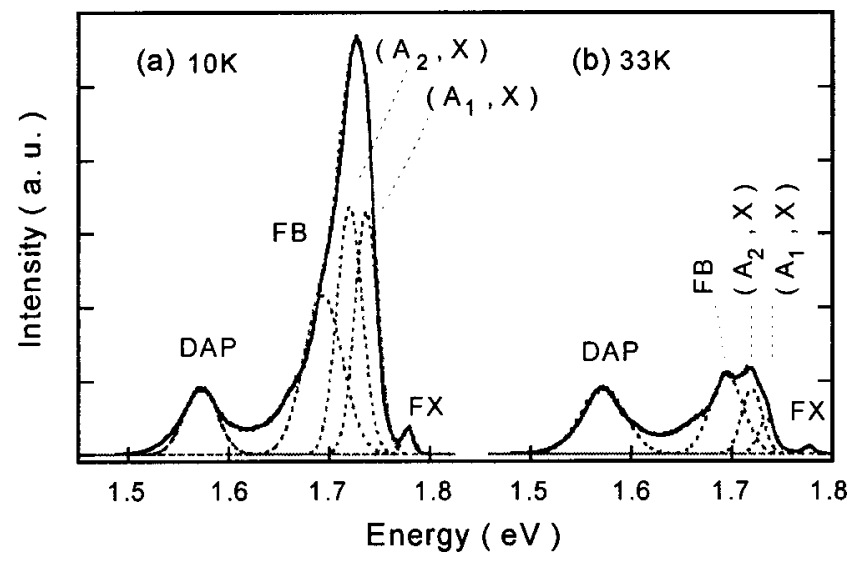

FIG. 9. Gaussian deconvolution of a PL emission spectrum in the 1.50-1.80 $\mathrm{eV}$ energy region for two different temperatures: (a) 10 and (b) $33 \mathrm{~K}$.

ties, increasing also the inelastic interaction between them. This interaction gives rise to electron-hole generation from free excitons, consequently increasing the number of free carriers at the expense of free exciton density. This favors the $F B$ transition as opposed to transitions due to bound exciton recombination.

The results of applying peak decomposition to the $B X$ complex band for two representative spectra can be seen in Figs. 9(a) and 9(b), where $1.730\left(A_{1}, X\right), 1.720\left(A_{2}, X\right)$, and $1.685 \mathrm{eV}(F B)$ are denoted by dashed lines together with the total spectrum (solid line). By performing this decomposition for a whole range of spectra as a function of the temperature and representing the integrated intensity versus $1 / k T$ for each individual component, like in Fig. 7, for the whole $B X$ band, we obtain activation energy values between 14 and $19 \mathrm{meV}$ for the $\left(A_{1}, X\right)$ and $\left(A_{2}, X\right)$ emissions and $35 \pm 5 \mathrm{meV}$ for the $F B$ transition.

The results indicate that the activation energy of bound exciton emission is close to the binding energy of the free exciton. As a result, thermal quenching of the bound exciton emission can be interpreted in terms of the dissociation of excitons. The exciton population decreases as the temperature increases because of thermal breaking of exciton binding but the $F B$ transition is less affected because it is related to free electrons. One may expect the activation energy of the $F B$ emission to be the ionization energy of the impurity

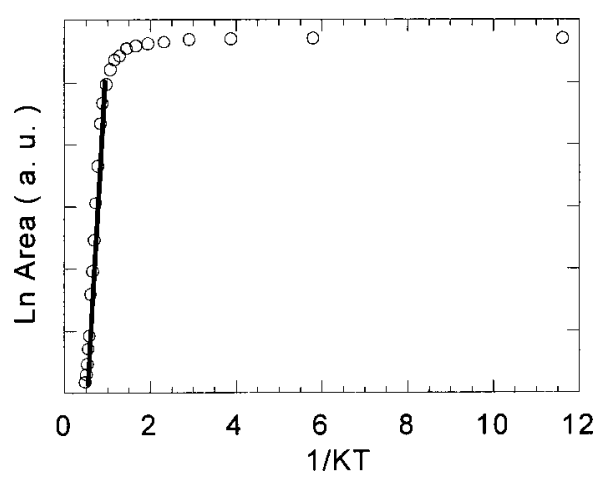

FIG. 10. Temperature dependence of the logarithmic emission-band area on the intensity of the DAP band. The slope of the fitted line gives the high temperature activation energy.
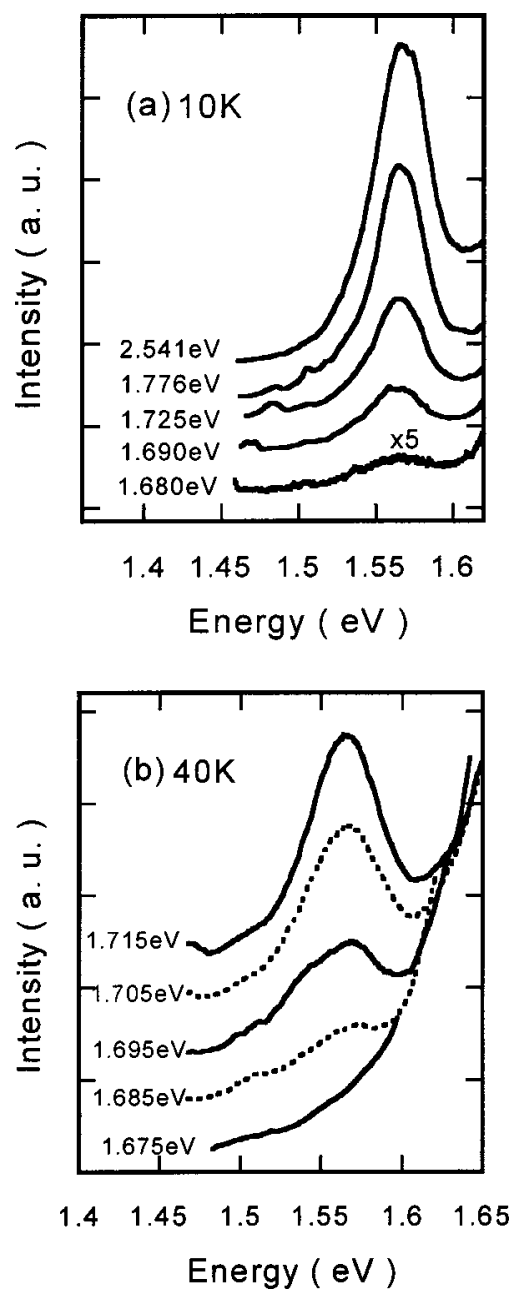

FIG. 11. Dependence of the emission spectrum intensity of the DAP band for different excitation energies at (a) 10 and (b) $40 \mathrm{~K}$.

involved in the recombination process, especially because when the thermal energy is of the order of this ionization energy, the level of impurity becomes full and the emission associated with it disappears. However in our case the value was too small to be the ionization energy of the impurity. Later we will come back to this in order to assign the activation energy to a given process.

\section{Donor-acceptor emission}

In the low energy zone a DAP broad band appears centered at $1.57 \mathrm{eV}$ and it extends through the $1.52-1.62 \mathrm{eV}$ range, with its shape and position changing slightly from sample to sample. As the temperature increases, this band also suffers a small blueshift before moving toward lower energies due to a decrease of the band gap. The high temperature activation energy of this DAP emission for all the samples investigated is $75 \pm 5 \mathrm{meV}$ (see Fig. 10), which is higher than the other emissions. Since the ionization energy of the donor levels is, in general, smaller than that of the acceptors, we will initially assign this energy to the donor ionization energy of the DAP transition.

The nature of this donor-acceptor pair band has been investigated using selective photoluminescence at different temperatures. The selective photoluminescence results also 


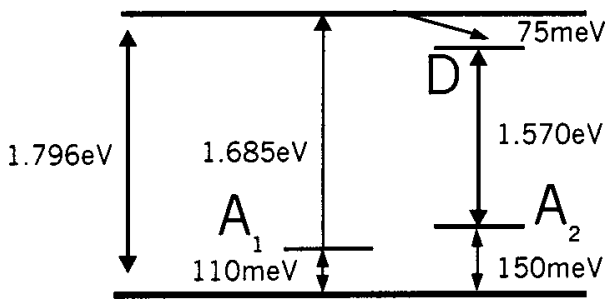

FIG. 12. DAP recombination diagram for excitation with light of $1.685 \mathrm{eV}$.

confirm the free-to-bound character of the $1.685 \mathrm{eV}$ emission. In Fig. 11(a) the DAP emission at $1.57 \mathrm{eV}$ is monitored for different excitation energies: the $2.54 \mathrm{eV}$ energy corresponds to the 488 line of the ion-argon laser, the $1.776 \mathrm{eV}$ energy corresponds to $F X$ recombination, the $1.725 \mathrm{eV}$ energy is associated with the bound exciton recombination energy, and the 1.690 and $1.680 \mathrm{eV}$ energies are those above and below the $F B$ emission, respectively. The results show that, when the sample is excited with energies lower than that of the $F B$ transition, there is no noticeable DAP emission. If the temperature is increased to $40 \mathrm{~K}$, the difference in intensity between the free-to-bound recombination and the excitonic recombination increases and the above behavior can be more clearly observed [Fig. 11(b)]. Selective photoluminescence results at $40 \mathrm{~K}$ confirm that the threshold energy for exciting the DAP emissions is around 1.685 $\pm 0.005 \mathrm{eV}$.

This threshold energy indicates the existence of an acceptor level in the band gap from which electrons are pumped to the conduction band. This acceptor level is located at $E_{\mathrm{I}}=E_{g}-1.685 \pm 0.005 \mathrm{eV}$. Therefore this acceptor level, which will be denoted $A_{1}$, is situated $110 \pm 5 \mathrm{meV}$ above the valence band. The observed $F B$ transition in the emission spectrum of the samples is due to the recombination of electrons from the conduction band into this acceptor level $A_{1}$ and, consequently, photons of $1.685 \pm 0.005 \mathrm{eV}$ are emitted.

The DAP emission has an activation energy of 75 $\pm 5 \mathrm{meV}$ which we have assigned as the ionization energy of the donor level. The ionization energy of the corresponding acceptor level, which will be denoted $A_{2}$, can be calculated from the relation $E_{g}=E_{\mathrm{DA}}+E_{D}+E_{A}$, giving a value of $150 \pm 5 \mathrm{meV}$ for $E_{A}$. The DAP transition takes place between the two levels shown in Fig. 12. Due to the existence of two acceptor levels, $A_{1}(110 \pm 5 \mathrm{meV})$ and $A_{2}(150$ $\pm 5 \mathrm{meV}$ ), we can expect bound exciton recombination $\left(A_{1}, X\right)$ and $\left(A_{2}, X\right)$, which corresponds to the 1.730 and $1.720 \mathrm{eV}$ emissions.

The exciton localization energy for the two recombination lines above can be calculated from the emission spectra, and a value of $38 \pm 3 \mathrm{meV}$ is obtained for the excitons bound to acceptor level $A_{1}$, with an ionization energy of $110 \mathrm{meV}$, and a value of $50 \pm 3 \mathrm{meV}$ for the excitons bound to acceptor level $A_{2}$, with $150 \mathrm{meV}$ ionization energy. If we apply the Haynes rule ${ }^{23}$ to the above emissions,

$$
E_{B X}=a+b^{*} E_{\mathrm{I}}
$$

and we set $a=0 \mathrm{meV}$, as is usually done, we obtain a value

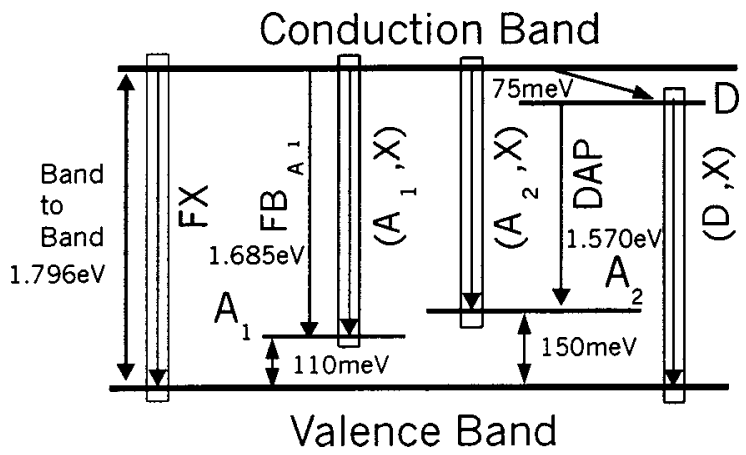

FIG. 13. Band gap levels and recombination observed in GaTe samples. The single arrows indicate free-to-bound recombination, the encircled arrowed lines indicate excitonic recombination, and the double arrows energy values.

of 0.3 for parameter $b$. This value, determined here for GaTe, is slightly higher than that for other semiconductors. ${ }^{23}$

The existence of a donor level at $75 \pm 5 \mathrm{meV}$ may produce bound exciton recombination at the centers of this level $(D, X)$. The related localization energy will be smaller than that of acceptor bound excitons, and the corresponding $(D$, $X)$ peak will be closer to the $F X$ peak than those of the $(A, X)$ recombination bands. Tentatively using the above calculated value of $b \cong 0.3$ in the Haynes' relation for the acceptor levels and ionization energy of $75 \mathrm{meV}$ for the donor level, the localization energy for recombination of an exciton at this donor level is around $20 \mathrm{meV}$, and the position of the $(D, X)$ peak is centered at $\hbar \omega=E_{F X}-E_{D X} \cong 1.76 \mathrm{eV}$. This emission is observable (Figs. 1 and 2) and it is the one that we initially called $D X$.

In Fig. 13 the electronic level distribution in the GaTe band gap is summarized together with the observed PL recombination. The activation energy of $35 \pm 5 \mathrm{meV}$ for the $F B$ $\left(A_{1}\right)$ emission at $1.685 \mathrm{eV}$ suggests that there exists a close energy level through which this emission deactivates. This energy of $35 \mathrm{meV}$ is very close to the difference in energy between the two acceptor levels, $A_{1}$ and $A_{2}$, suggesting that the $F B\left(A_{1}\right)$ transition deactivates through close level $A_{2}$ when the thermal energy is of the order of this difference.

Due to the existence of the $A_{2}$ level, a free-to-bound transition has to be expected around $E g-150 \mathrm{meV}$, that is, at $1.65 \mathrm{eV}$. The presence of this emission could be the reason for the difference between the total PL emission and that obtained by superposition of the single emission lines described here (Fig. 9).

By comparing the results obtained in this work with those obtained in previous studies, ${ }^{15,16,18}$ the existence of an acceptor level at $150 \mathrm{meV}$ can be confirmed. This level agrees with that previously obtained by Hall effect measurements. ${ }^{17}$ The DAP recombination observed in our work at $1.57 \mathrm{eV}$ is due to recombination to the acceptor level at $150 \mathrm{meV}$ from a donor level, as initially mentioned by Shigetomi et al. ${ }^{16}$ However, our results do not confirm the mechanism proposed by Guder et al. ${ }^{18}$ which stated that the DAP band is due to recombination from a donor level at 170 $\mathrm{meV}$ to an acceptor level at $56 \mathrm{meV}$. 


\section{Assignment of the band gap levels}

The results of inductively couple plasma-optical emission spectroscopy for several samples show the presence of $\mathrm{Na}$ and $\mathrm{Si}$ in a quantity of about $1-50 \mathrm{ppm}$. Some samples also contain $\mathrm{Li}$ at small quantities of around $10 \mathrm{ppm}$. Other elements such as $\mathrm{Cu}, \mathrm{Ag}, \mathrm{Al}, \mathrm{Au}, \mathrm{Cl}, \mathrm{P}$, and As were below the detection limit of the equipment $(0.1 \mathrm{ppm})$. Scanning electron microscopy analysis of the surface of the samples shows small inclusions in some of them, which were analyzed by x-ray energy dispersion spectroscopy. The results show that $\mathrm{Si}$ impurities are present in the inclusions.

$\mathrm{Li}$ and $\mathrm{Na}$ are $\mathrm{Ga}$ substitutional impurities and they can form acceptor centers. With respect to the Si present in the localized inclusions, we can assume that some atoms are present in the GaTe matrix. They can be in a substitutional position of $\mathrm{Ga}$ and behave like donor impurities.

As a result of the above results, we can tentatively assign the $A_{1}(110 \mathrm{meV})$ and the deeper $A_{2}(150 \mathrm{meV})$ acceptor levels to $\mathrm{Li}$ and $\mathrm{Na}$ atoms, although $\mathrm{Ga}$ vacancies or their complexes could be involved in these levels. Si as a substitutional impurity on Ga sites could be responsible for the donor level detected at $75 \pm 5 \mathrm{meV}$. The possible sources of impurity contamination in the case of $\mathrm{Na}$ and $\mathrm{Li}$ could be the handling process, even though extreme care was taken during the preparation and the growth process of the samples. In the case of $\mathrm{Si}$, the contamination could arise from the $\mathrm{Si}$ content of the quartz ampoules in which the crystal was grown.

\section{CONCLUSIONS}

Unintentionately doped high quality crystals of the laminar semiconductor $p$-GaTe were studied. The variation in the gap was obtained by direct PL measurements over the 10$200 \mathrm{~K}$ range and fitting of the experimental values using the model of O'Donnell and Chen ${ }^{19}$ has allowed extrapolation of the energy gap at higher temperatures. The results indicate that the phonons involved in the variation of the band gap are LO phonons with energy of $14 \mathrm{meV}$. The existence of an acceptor level at $150 \pm 5 \mathrm{meV}$ was confirmed and it is assumed to be responsible for the observed DAP recombination resulting from impurities. The center band in GaTe observed by Taylor and Ryan ${ }^{15}$ and by Guder et al. ${ }^{18}$ is a band in which different emissions can coexist: the recombination of excitons of at least one donor level at $75 \pm 5 \mathrm{meV}$, two acceptor levels at $110 \pm 5$ and $150 \pm 5 \mathrm{meV}$, and free-tobound recombination. A value of 0.3 for the $b$ parameter used in Haynes' rule was obtained that can be used in future work to determine the ionization energy of acceptors levels in which excitons recombine. Si is present as an impurity in the samples grown and it could be responsible for the donor level. This latter impurity may have been added to the sample by the quartz ampoules used for the sample growth process.

\section{ACKNOWLEDGMENT}

This work was partially supported by the Spanish Comisión Interministerial para Ciencia y Tecnología under Grant Nos. MAT98-0975, 1FD97-0086, and MAT2001-2920.

${ }^{1}$ W. B. Pearson, Acta Crystallogr. 17, 1 (1964).

${ }^{2}$ M. Julien-Pouzol, S. Jaumes, M. Guitard, and F. Alapini, Acta Crystallogr., Sect. B: Struct. Crystallogr. Cryst. Chem. B35, 2848 (1979).

${ }^{3}$ M. Schluter, J. Camassel, S. Khon, J. P. Voichovsky, Y. R. Shen, and M. L. Cohen, Phys. Rev. B 13, 3534 (1976).

${ }^{4}$ V. Augelli, C. Manfredotti, R. Murri, R. Piccolo, A. Rizzo, and L. Vasenelli, Solid State Commun. 21, 575 (1977).

${ }^{5}$ L. Gouskov and A. Gouskov, Solid State Commun. 28, 99 (1978).

${ }^{6}$ J. F. Sanchez-Royo, A. Segura, and V. Muñoz, Phys. Status Solidi A 151, 257 (1995).

${ }^{7}$ J. Pellicer-Porres, A. Segura, A. San Miguel, and V. Muñoz, Phys. Status Solidi A 211, 389 (1998).

${ }^{8}$ J. Camassel, P. Merle, and H. Mathieu, Physica B 99, 309 (1980).

${ }^{9}$ J. Z. Wan, J. L. Brebner, R. Leonelli, and J. T. Graham, Phys. Rev. B 46, 1468 (1992)

${ }^{10}$ J. Z. Wan, J. L. Brebner, R. Leonelli, G. Zhao, and J. T. Graham, Phys. Rev. B 48, 5197 (1993).

${ }^{11}$ J. Z. Wan, J. L. Brebner, and R. Leonelli, Phys. Rev. B 52, 16561 (1995).

${ }^{12}$ J. Z. Wan and R. Leonelli, Phys. Lett. A 219, 345 (1996).

${ }^{13}$ J. Z. Wan, Fred H. Pollack, J. L. Brebner, and R. Leonelli, Solid State Commun. 102, 17 (1997).

${ }^{14}$ A. Syouji, A. Yamamoto, and T. Goto, Phys. Rev. B 60, 15519 (1999).

${ }^{15}$ R. A. Taylor and J. F. Ryan, J. Phys. C 20, 6175 (1987).

${ }^{16}$ S. Shigetomi, T. Ikari, and H. Nishimura, J. Lumin. 78, 117 (1998).

${ }^{17}$ G. Fischer and J. L. Brebner, J. Phys. Chem. Solids 23, 1363 (1962).

${ }^{18}$ H. S. Guder, B. Abay, H. Efeoglu, and Y. K. Yogurtcu, J. Lumin. 93, 243 (2001)

${ }^{19}$ K. P. O’Donnell and X. Chen, Appl. Phys. Lett. 58, 2924 (1991).

${ }^{20}$ Y. P. Varnish, Physica (Amsterdam) 34, 149 (1967).

${ }^{21}$ J. C. Irwin, B. P. Clayman, and D. G. Mead, Phys. Rev. B 19, 2099 (1979).

${ }^{22}$ J. Krustok, H. Collan, and K. Hjelt, J. Appl. Phys. 81, 1442 (1997).

${ }^{23}$ J. R. Haynes, Phys. Rev. Lett. 4, 361 (1960). 